\title{
Security and Integrity of a Distributed File Storage in a Virtual Environment
}

\author{
Gaspare Sala $^{\dagger} \quad$ Daniele Sgandurra $^{\dagger} \quad$ Fabrizio Baiardi* $^{*}$ \\ ${ }^{\dagger}$ Dipartimento di Informatica, Università di Pisa \\ *Polo G. Marconi - La Spezia, Università di Pisa \\ \{sala, daniele, baiardi\}@di.unipi.it
}

\begin{abstract}
Virtual environment Secure File System (VSFS) is a software architecture for secure file sharing among applications with different trust levels that consists of a set of interconnected virtual machines (VMs). Application VMs (APP-VMs) run the application processes that transparently access remote shared files hosted by file system VMs (FS-VMs). Each FS-VM implements a Mandatory Access Control (MAC) security policy to control file sharing. To define and enforce this policy, VSFS uses SELinux. Each APP-VM is labeled with a security context paired with the IP address of the VM. FS-VMs use this context to check access rights of the $A P P-V M s$ with respect to the requested files and operations. A third set of VMs, the administrative VMs (A-VMs), provides assurance about the integrity of the FS-VMs and implements anti-spoofing techniques to authenticate each file request sent by the APP-VMs.

After describing the overall architecture, we discuss the security and performance results of a first prototype. These first results show that the overhead due to mandatory access control is fairly acceptable.
\end{abstract}

Keywords: virtual machines, introspection, trust level, network file system, mandatory access control

\section{Introduction}

Secure file sharing among applications with different trust levels is a fundamental requirement for Web Services or $\mathrm{P} 2 \mathrm{P}$ applications, because users are willing to supply and share their data only if they receive some assurance on the description and enforcement of the security policy that controls the sharing. The complexity of the resulting system increases because the policy has to be enforced in a transparent way to both the users and the applications.

This paper presents Virtual environment Secure File System (VSFS), a software architecture that supports file sharing among applications executed by users with different trust levels, where an application may contaminate other ones through shared files [32]. To prevent contamination, a security policy for each file system constrains the files each user can access and the operations that can be invoked.

VSFS is based upon virtual machines (VMs) [4, 5, 11]. $\mathrm{A} V \mathrm{VM}$ is an execution environment created by a virtualization technology such as Xen or VMWare that introduces a new layer into the computer architecture, namely the virtual machine monitor (VMM) [14]. This is a thin software layer in-between the OS layer and the hardware/firmware one that creates, manages and monitors the VMs that run on the VMM itself. In this way, the same physical machine can run several OSes, each on a distinct VM.

VSFS includes distinct kinds of VMs, namely APPVMs that run some application processes, FS-VMs that export shared file systems and A-VMs that perform administrative tasks. The requests of an application to access a shared file are transparently intercepted on an APP-VM and delivered to the proper FS-VM, which implements the security policy defined by the owner of the file system it exports.

To increase system security, VSFS adopts virtual machine introspection (VMI) [13] to evaluate a set of consistency checks on kernel data structures in the memory of the FS-VMs to detect attacks against them. VMI exploits VMM direct access to the physical memory to gather all the information it needs to rebuild some kernel data structures of a VM starting from the 
raw values in memory. In the case of a FS-VM, the complexity of the resulting security checks on these kernel data structures is not high because the FS-VM only runs a minimal kernel with just a few services and a file server.

In the current prototype, FS-VMs run SecurityEnhanced Linux (SELinux) [3, 17, 18] to support both discretionary (DAC) and mandatory access control (MAC) policies and to enforce the security policy in a centralized way.

The rest of the paper is organized as follows. Sect. 2 describes the overall architecture of VSFS and introduces the application and the threat models we assume. Sect. 3 discusses the current implementation. Sect. 4 and 5 present, respectively, a first evaluation of VSFS from the security point of view and from the performance one. Sect. 6 discusses some related works. Finally, Sect. 7 draws a first set of conclusions and outlines future developments.

\section{Overall Architecture and Threat Model}

This section describes the architecture of VSFS and the model that defines the applications of interest, the threats and the attacks against the applications.

\subsection{VSFS Architecture and Applications}

The application model of interest consists of a set of application processes executed by several users, where each process $P$ can access some files in one or more shared file systems, denoted as $F S_{1}(P), \ldots, F S_{n}(P)$. We assume that $T(P), P$ trust level, is known and that it also holds for all the processes spawned by $P$.

To execute the application processes and export the file systems, the application model introduces a cluster of VMs mapped onto a, smaller, cluster of physical machines. Each physical machine runs a VMM that multiplexes its physical resources and guarantees confinement among the VMs it supports. The cluster of VMs includes three disjoint sets of VMs:

1. APP-VMs that run the application processes;

2. FS-VMs, each exporting a file system shared among the application processes.

3. one A-VM for each physical node, to set up and manage the cluster of VMs.
Each FS-VM implements a highly secure storage through a file sharing server (FSS) module configured according to the security policy defined for the corresponding file system. Each APP-VM runs a file sharing client (FSC) module that acts on behalf of the application processes and interacts with the proper FSS module, so that application processes can access any files unaware of the type and the location of the file system.

The mapping of applications and file systems onto the physical cluster is implemented in two steps:

1. the first one maps the application processes onto APP-VMs and each shared file system onto a distinct FS-VM;

2. the second step maps all the VMs, APP-VMs and FS-VMs, onto the physical machines to balance the computational load on the machines and the communication load on the interconnection structure.

The mapping in the first step is static, i.e. an application process cannot migrate from an APP-VM to another one, because we assume that two processes executed by users with distinct security requirements, i.e. with different trust levels, should not be mapped onto the same APP-VM. The goal of this step is to minimize the sharing among the processes. On the other hand, the mapping in the second step is dynamic and an A$\mathrm{VM}$ can migrate at runtime an APP-VM or a FS-VM to a different physical node (see Fig. 1).

To show how an application process accesses a file, consider an application process $P$ on $A P P-V M(P)$, which locally mounts any shared file system $F S_{i}(P)$ exported by $F S-V M_{i}(P)$. While the local file system of $A P P-V M(P)$ handles any operation on $P$ private files, any request of $P$ to access a file in $F S_{i}(P)$ is trapped and transferred to the $F S C$ module of $A P P$ $V M(P)$ that transmits it to the $F S S$ module on $F S$ $V M_{i}(P)$. This module checks the request and, if it satisfies the security policy of $F S_{i}(P)$, it serves the request and transfers the result to $F S C$. $P$ is unaware that it is accessing a file on a distinct VM.

If we consider file sharing as a specific case of resource sharing, most of the previous concepts applies to the more general context too. 


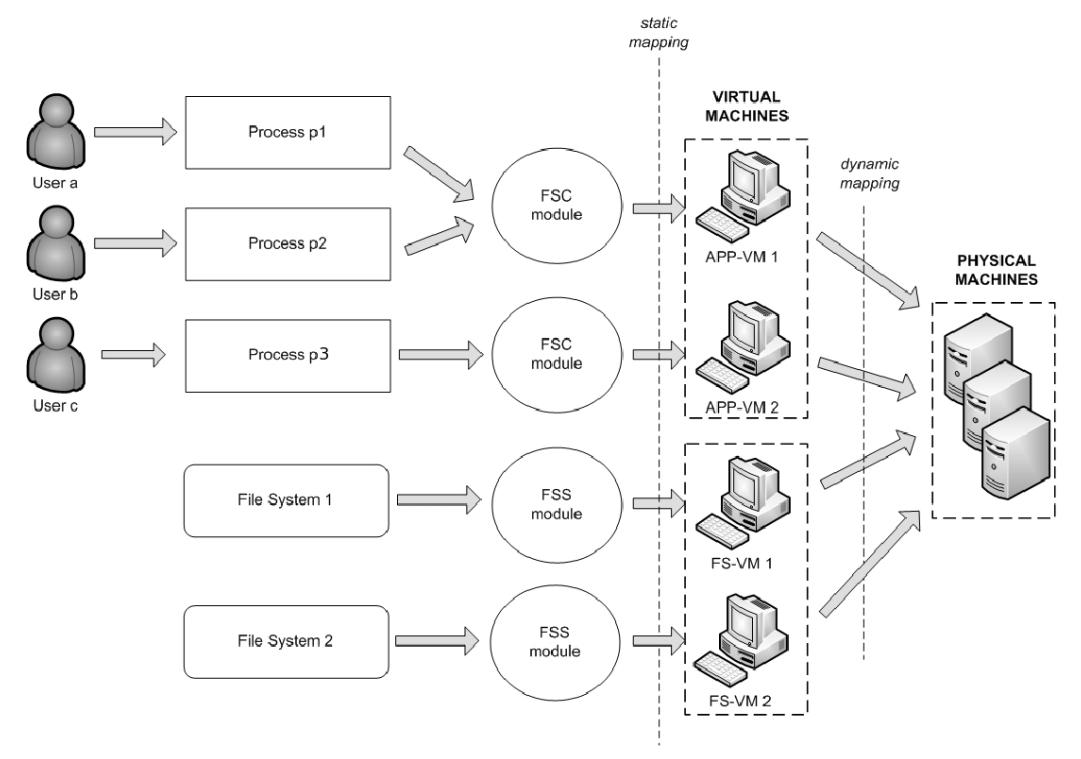

Figure 1. Application Model.

\subsection{Threat Model}

The threat model we assume is focused on the attacks of some application processes against some other ones. This assumes that each application process may act on behalf of an unauthorized user to implement attacks with the goal of accessing some shared resources, files in the considered model. The attacks may be implemented either by a malicious application or by malware code that replaces the application code as a result of a previous attack.

We also assume that communications among the physical nodes cannot be forged or spoofed, because no direct attack is implemented against the physical network infrastructure. On the other hand, the virtual connections among the VMs on the same physical node can be attacked. To prevent these attacks, the IP address of each VM is statically assigned and known and the consistency of communications among the VMs has to be guaranteed by preventing a VM to spoof an address. A further assumption underlying the adopted threat model is that the VMM, the FS-VMs and the A-VMs are all trusted, i.e. they belong to the Trusted Computing Base (TCB).

As an example, this threat model describes in a realistic way a trusted intranet executing a set of untrusted applications, where each application can implement attacks to export some information it illegally accesses. The intranet connections are trusted because the information they transfer among the physical nodes cannot be sniffed or altered. The threat model is generalized in Sect. 7.

\subsection{Security Policy: Implementation}

As previously described, each physical node runs a VMM that supports multiple APP-VMs and FS-VMs. Each APP-VM executes a set of applications by users with the same trust level. From a security perspective, the VMM guarantees the confinement among the resources of distinct VMs.

To implement in a transparent way the security policy that controls file sharing, each APP-VM executes a FSC module that remotely accesses a FSS module on a FS-VM. In turn, the FSS module delegates the security policy description and enforcement to a $M A C$ based Security (MAC-S) module, which guarantees the fulfilment of critical security requirements, such as the integrity and the confidentiality of the shared storage. The adoption of a MAC-S module enables VSFS to support a large set of MAC or DAC class policies on the shared storage and forces users to respect the adopted security policy. As an example, MAC allows VSFS to reduce the privileges associated to the superuser on the APP-VMs and to minimize the impact on the shared storage due to a flawed or malicious appli- 
cation process on an APP-VM.

Every file request that the FSC module forwards to a FSS module includes the IP address of the APP-VM that produces the request. The MAC-S module uses this information to protect the shared storage, through a default-deny approach where an application can only access those files for which the current policy grants an authorization. To pair a protection domain with each APP-VM, the MAC-S module identifies the APP-VM through the source IP address in the request and labels the APP-VM with a security context according to the set of privileges associated with the trust level paired with the IP address. Therefore, if the security policy is parametric with respect to the APP-VM trust level, the user that executes an application process inherits the protection domain of the APP-VM that runs the process. In this way, all the users of an APP-VM can access the same set of files with the same privileges, whereas users on APP-VMs with different trust levels cannot access the same set of files with the same privileges. Hence, the granularity level of the overall security policy can be tuned by updating the number of APP-VMs.

\subsection{Security Policy: Assurance}

VSFS integrates Psyco-Virt [7], an introspection based IDS to detect attacks against the VMs. PsycoVirt requires a further VM for each node, the introspection $V M$, which analyzes the memory of VMs onto the same node through virtual machine introspection to discover attacks against the kernel of these VMs. VSFS assumes that both an APP-VM and a FS-VM may run some agents to detect intrusions against the local OS or the application processes. All the VMs on the same physical node are connected to the introspection VM through a dedicated control network that delivers the agent alerts to the introspection VM. A typical application of introspection is the protection of the memory areas storing the code of the OS kernel. A hash value of each area is periodically computed to detect attacks against the code it stores.

$\mathrm{VM}$ introspection is even more powerful when applied to a FS-VM because this VM runs a fixed set of processes, which use a fixed set of storage resources and so on. Because of these constraints, the introspection VM can apply to any FS-VM a well known num- ber of consistency checks.

The introspection VM increases the overall assurance by:

- checking the integrity of the file storage, i.e. of the FS-VM;

- implementing checks on spoofed IP packets.

The first task requires that this VM applies virtual machine introspection to check at least the integrity of the OS kernel in the FS-VM. Virtual machine introspection can also check the integrity of any critical process in an APP-VM. The existence and the numbers of these checks strongly depend upon the required degree of security, the threats that are considered and the computational load on each APP-VM. However, since applications running inside APP-VMs cannot be trusted, an introspection VM can fully protect the integrity of the FS-VMs only. This also implies that further security mechanisms, such as spoofing prevention, are not effective if executed inside an APP-VM. In some cases, however, it may be useful to enforce further protection controls on the APP-VMs themselves, such as consistency checks and intrusion detection analysis, to add redundancy checks and reduce the probability of successful attacks.

The ability of applying introspection is a further advantage of a VM-based architecture because if the OS and the applications are not executed inside a VM, then introspection requires a proper hardware/firmware support [22].

To detect spoofed packets, IP addresses are statically assigned to the APP-VMs, so that the introspection VM can check if the source IP address of every packet is the same of the APP-VM that generates the packet.

\section{Implementation of VSFS}

A first prototype of VSFS (see Fig. 2) is based on Xen [6], an open source virtualization technology developed at the University of Cambridge Computer Laboratory. Xen is integrated into the VSFS architecture to create the virtual environments that run the applications and export the shared file systems.

The main implementation choices of the current prototype are: 


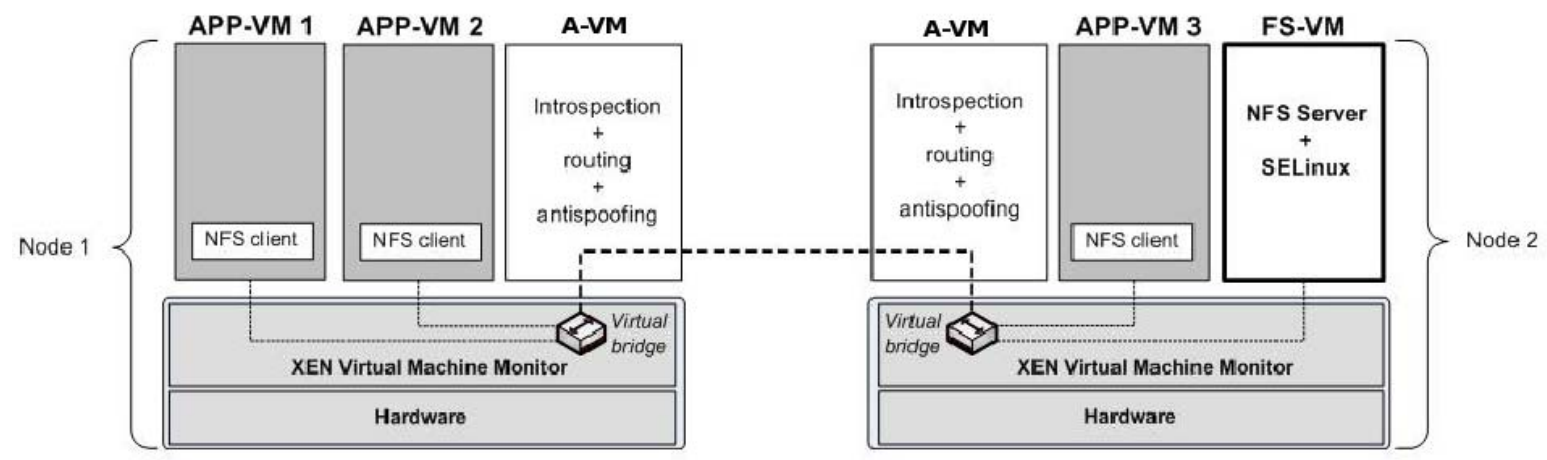

Figure 2. VSFS Architecture.

- the development of modules to describe in a centralized way a distributed security policy to handle file requests, and to force users to respect their roles when accessing a file storage. These modules are based upon the NFSv3 service [9] and SELinux;

- to simplify the overall architecture, the task of the introspection $\mathrm{VM}$ is assigned to the $\mathrm{A}-\mathrm{VM}$;

- all the VMs mapped onto the same node are connected to the network through a virtual bridge.

NFSv3 Overview. The NFS service exploits a client-server architecture to implement a distributed file system by exporting to the clients one or more directories of the shared file system. According to the general model previously described, each APP-VM executes one and only one NFSv3 client, i.e. the FSC module, and every FS-VM executes both a NFSv3 server, i.e. the FSS module, and a SELinux module, i.e. the MAC-S module.

Currently, NFS servers exploit the information in each RPC request generated by the client to authorize or deny access to the shared files, according to the server OS DAC class policies. Since, according to our threat model, clients represent a source of untrusted information, NFS has to be modified because it essentially trusts the client machines, and it enables an attacker to maliciously impersonate a legitimate user on an APP-VM with little effort [20].

SELinux Overview. SELinux implements MAC policies through a combination of type enforcement
(TE), role-based access control (RBAC) and Identitybased Access Control (IBAC). The TE model assigns types to every OS objects, such as files, processes, and network connections. In this way, the security policy can define the rules governing the interactions among OS objects, by implementing a fine-grained access control that satisfies the least privilege principle [25]. SELinux is based upon the Linux Security Modules (LSM) [26, 28], a patch for the Linux kernel that inserts both security fields into kernel data structures and calls to specific hooks into security-critical kernel operations to manage the security fields and to implement access control.

When the SELinux policy is being configured, an administrator can label every kernel component with a security context. Processes, identified by a domain, are the subjects of the SELinux policy. At runtime, the security policy can pair the subject with its privileges to grant or deny access to system objects according to the requested operation. The policy description specifies both the programs a process can execute and the legal domain transitions.

The idea of integrating NFS with SELinux stems from the need to centrally control client accesses to the shared files and to assign distinct privileges to each APP-VM, leveraging the SELinux flexibility to describe MAC policies.

\subsection{Modifications to NFS and SELinux}

To develop VSFS we have modified the Linux kernel to enable a FS-VM to exploit a simpler resource sharing management and a fine-grained access control mechanism. A compile-time option enables the ad- 


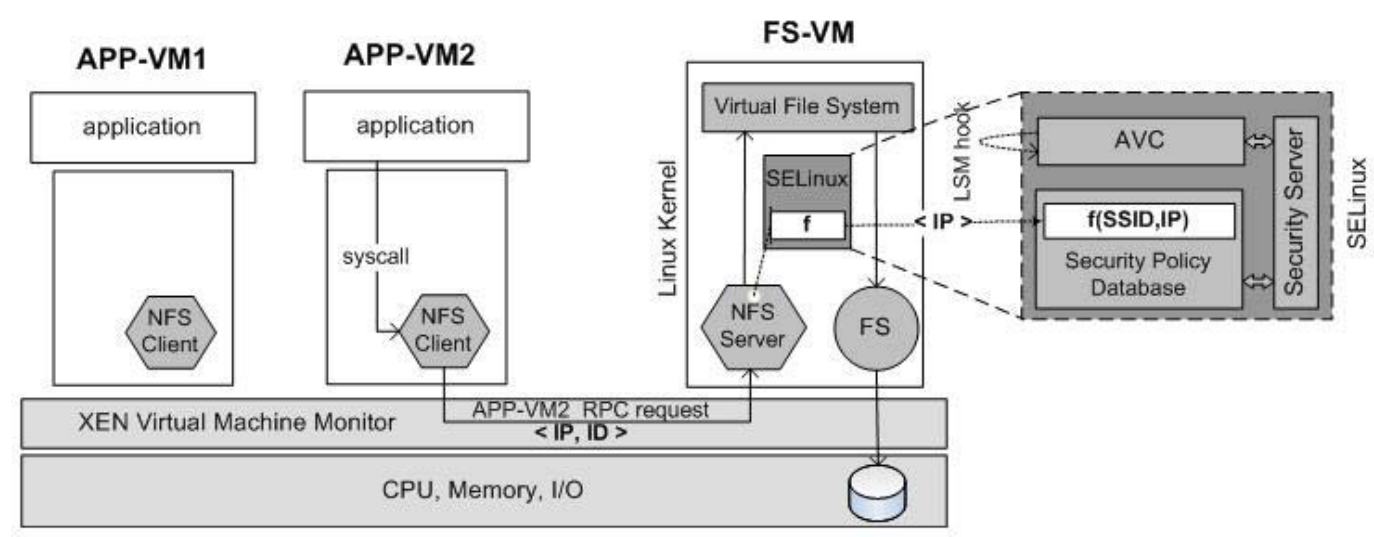

Figure 3. FS-VM.

ministrator to configure the kernel of the FS-VM to integrate SELinux and NFS. On the other hand, no modifications are required on the APP-VMs.

Through the modified SELinux labeling and access rules, FS-VM administrators can manage, from a single central point, the indirect accesses of an NFS client to the shared file systems. As an example, an administrator can pair each NFS client with a security context and, if proper privileges are assigned to this context, VSFS can satisfy the least privilege principle without sacrificing transparency.

Moreover, since the A-VM can identify the IP address of each APP-VM in a reliable way, FS-VM considers the APP-VM that generated the file request as the real subject of the current security policy.

NFS Client Subject. SELinux labeling and access rules have been changed to introduce a new subject corresponding to the NFS client and to define all the operations it can invoke. As a consequence, we have extended the SELinux network object, called node [19], by adding into the corresponding object class the operations executed by the NFS server on behalf of NFS clients, such as read, write and create files or directories. In general, nodes are used to control the network traffic, i.e. to grant or deny a process the permissions to exchange data with a specific IP address through the network interfaces, and are associated with an IP address and a net-mask through the nodecon SELinux syntax statement.

These modifications allow VSFS to define a distinct protection domain for each NFS client and to dynami- cally pair the NFS server process with the security context of the NFS client requesting the file.

NFS Request. To properly describe the corresponding updates to the kernel modules, we consider the flow of a request from a NFS client to the NFS server and show how the data structures have been changed and where the modified functions are invoked.

SELinux uses some data structures to store runtime security information about the kernel objects, such as tasks, i-nodes and files. The main structure, task_security_struct, denoted in the following as TaskSecStruct, stores security information about the running processes.

NfsSid is a new field of TaskSecStruct we have added to represent the security identifier bound to the node type. This field is paired with the IP address and the net-mask of the NFS client that is trying to access the shared file. Every time the NFS server processes a request, the RPC service calls a new SELinux function, called $f$ in Fig. 3, that maps the NFS server SID (SSID) and the NFS client IP address into a SID according to the SELinux Security Policy Database, i.e. the database that stores the current SELinux policy. If the current security policy pairs the requesting IP address with a node type, $f$ returns the corresponding SID and the related security context, otherwise it returns a default unprivileged SID. Before the NFS server invokes the system call on the file system, the NFS client SID is copied into the NfsSid field of SecTaskStruct of the NFS daemon process servicing the request. Later, when the NFS server invokes 


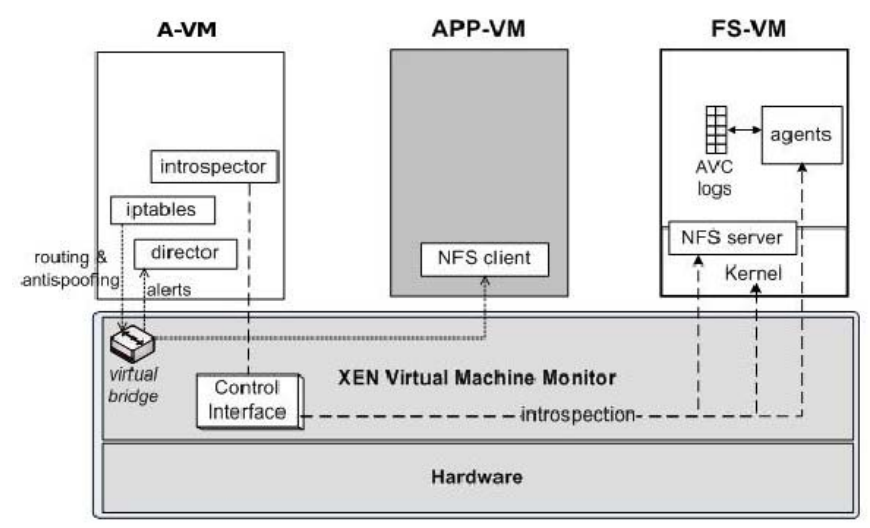

Figure 4. Administrative VM.

the system call to access the shared file system on behalf of NFS clients, the kernel triggers a LSM hook to delegate security controls to SELinux.

LSM Hook Modifications. The appropriate LSM hooks have been modified to enforce access controls on the operations a subject can invoke. The controls are applied when the NFS server:

- uses a capability;

- updates an i-node;

- updates a file;

- creates or removes a file, a directory, a link to a file or to a directory;

- renames a file or a directory;

- operates on the file system (super-block).

We have modified all these hooks so that two cases are considered. If the NFS server task is paired with the SID relative to the IP address of the requesting NFS client, then the SELinux Security Server applies the current security policy by considering the NfsSid as the policy subject. If, instead, an unprivileged default SID relative to the node type is assigned to the SecTaskstruct, then the subject of the SELinux controls is the NFS server daemon process. After the kernel has handled the system call, the NfsSid field is reset to a default value corresponding to an unprivileged domain. In both cases, the Security Server authorizes or denies file access according to the current security policy. In this way, NFS clients own some privileges on the remote file system exported by the FS-VM just for the time interval when the kernel serves client requests on a shared file.

\subsection{Administrative Virtual Machine}

The A-VM (see Fig. 4) simplifies the configuration of the overall system by enabling the administrator to start/stop/migrate a VM on demand. It also manages the connection among the various physical nodes and prevents IP spoofing on the virtual bridge, and finally it protects the kernel integrity of the FS-VMs.

Introspection. The A-VM exploits virtual machine introspection to monitor the FS-VMs by checking the integrity of the kernel code and of kernel critical data structures. These checks guarantee the integrity of all the processes spawned by the NFS daemons, such as portmapper, rpcd.nfsd and rpcd. mountd, as well as of basic kernel functionalities.

As an example, the A-VM checks the pages storing the following areas of the OS kernel:

- the area containing the kernel code, from the address_text to_etext;

- the system call dispatch table, stored in the sys_call_table array;

- the interrupt descriptor table, stored in the idt_table table. 
Moreover, the kernel of FS-VMs is extended with further security functions to compute at regular intervals the hashes of the text area of critical processes of the FS-VMs, such as those spawned by the agents. Further security checks on the FS-VMs are also implemented by SELinux.

Agents. Distinct agents may be introduced to detect attacks against a VM according to the VM trust level. On an APP-VM, fairly classical agents can be used and the kernel essentially checks that the programs and the data of these agents have not been subverted. On a FS-VM, the set of checks can be more effective due to the tighter constraints that this VM satisfies. As an example, this VM only runs the processes that serve requests from a well known set of APP-VMs, and therefore no other process should be created and only requests from the proper APP-VMs should be served. In this section, we will only focus on agents that may be instantiated on the FS-VMs.

A FS-VM agent scans the log file looking for AVC denial messages. By default, SELinux logs access vector cache (AVC) denied messages in /var/log/messages. This agent sends an alert to the A-VM each time the denied field of the AVC log matches any security critical operation, for example when a node type tries to execute an operation not defined in the SELinux policy. Then, the A-VM can freeze the APP-VM that has attempted the operation. Further agents can be easily added to a FS-VM, such as one that checks the amount of free disk space, or the number of open files.

Anti-spoofing. To implement the anti-spoofing control on the virtual bridge, the A-VM kernel is compiled by enabling the following options:

- CONFIG_NETFILTER_XT_MATCH_PHYSDEV

- CONFIG_BRIDGE_NETFILTER

- CONFIG_NETFILTER_NETLINK

- CONFIG_NETFILTER_XTABLES

- CONFIG_BRIDGE

Furthermore, we have defined an iptables [2] FORWARD rule for every possible legal connection between two VMs implemented through the virtual bridge. Each of these rules is defined in terms of the static IP address bound to the virtual interface assigned to each APP-VM. Every packet with a spoofed source IP address is dropped and logged.

\section{Security Results}

This section shows a first set of security results. In particular, we show what happens when an APP-VM forges an IP packet with a spoofed IP address, or it attempts an illegal operation on a file. Since the VSFS architecture is not focused on NFS security, we do not detail the detection of attacks that exploit NFS vulnerabilities, such as buffer overflows. However, VSFS can prevent most of these attacks, such as the execution of code in the stack or the injection of instructions into the text area of a process, because it integrates SELinux and virtual machine introspection.

A first security test considers spoofing. To prevent it, the A-VM exploits IPTABLES forward rules, such as the following one:

/sbin/iptables -A FORWARD - s 10.0.0.103 -m physdev --physdev-in tapl -j ACCEPT

This rule states that the virtual interface $\operatorname{tap} 1$, bound to a specific APP-VM, can only forward packets with 10.0.0.103 as the source IP address. If the IP address of the interface is set to 10.0.0.109, each time the APPVM tries to exchange packets through this interface, IPTABLES drops the packets and for each packet it creates an entry in the log file, such as the following one:

May 22 15:24:17 192.168.1.103 kernel:

[FORWARD DROP] : IN=xenbro OUT=xenbro

PHYSIN=tap1 PHYSOUT=tap0 SRC=10.0.0.109

$\mathrm{DST}=10.1 .0 .102 \mathrm{LEN}=84 \mathrm{TOS}=0 \times 00 \quad \mathrm{PREC}=0 \times 00$

$\mathrm{TTL}=64 \mathrm{ID}=0$ DF $\mathrm{PROTO}=\mathrm{ICMP} \quad \mathrm{TYPE}=8 \quad \mathrm{CODE}=0$

$\mathrm{ID}=24329 \mathrm{SEQ}=1$

After updating the log, the A-VM stops the execution of the APP-VM that generated the spoofed IP packet.

Our second security test considers an application process that attempts an illegal operation on a shared file. We have added new rules in the SELinux policy configuration file, such as the following ones: 
nodecon 10.0.0.101 255.255.255.255

system_u : object_r : node_mv101_nfs_t

...

allow nfsd_t node_mv101_nfs_t:node $\{$ tcp_recv

tcp_send $\}$;

...

allow node_mv101_nfs_t dir_mv101_roperm: dir

r_dir_perms;

The first rule pairs the security context called system_u:object_r:node_mv101_nfs_t with the IP address 10.0.0.101. The second one enables the $n f s d$ task, labeled with the domain $n f s d \_t$, to invoke TCP operations to interact with the node type node_mv101_nfs_t. The last access rule allows this node to read and set attributes of the files in the directory labeled with dir_mv101_roperm.

When the $n f$ sd task, paired with the SID relative to the IP address of the requesting APP-VM, attempts an illegal operation, the corresponding FS-VM logs the following AVC denial message to inform that it has denied a write operation on a file:

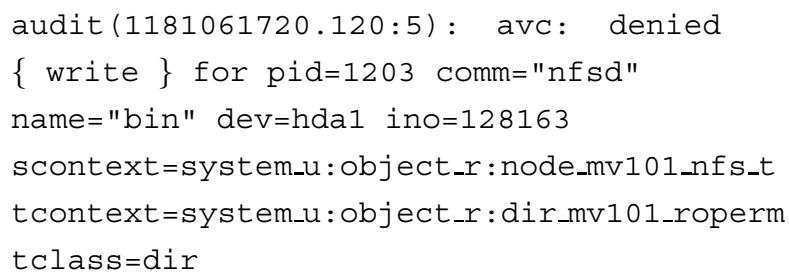

In this case, an agent on the FS-VM sends an alert to the A-VM, which simply displays the log message on the system console. In turn, the A-VM administrator can freeze the execution of the APP-VM that produced the denied request.

\section{Performance Results}

We used the IOzone Filesystem Benchmark [1] to run NFS performance tests. To evaluate the relative overhead due to the enforcement of the security policy, the benchmark has been executed in the following cases:

- on an APP-VM on the same physical node of the FS-VM with SELinux disabled;
- as the previous one with SELinux enabled;

- on an APP-VM on a different physical node of the FS-VM that has SELinux disabled;

- as the previous one with SELinux enabled.

Tab. 1 describes the configuration of the machines running the performance tests.

\begin{tabular}{|l|l|}
\hline CPU & Intel Core Duo T2300 1.66GHz \\
VMM & Xen 3.0.2 \\
A-VM & Fedora 6 Linux 2.6.19-xen, 512MB memory \\
FS-VM & Debian Linux 2.6.16.1-xen, 128MB memory \\
APP-VM & Debian Linux 2.6.19-xen, 64MB memory \\
Network & Ethernet 100Mb/s \\
\hline
\end{tabular}

Table 1. Test Configuration

Fig. 5(b) and 5(a) show, respectively, the average and max throughput of the read and write tests. The overhead due to SELinux in the write test is about $11 \%$ on the same node and negligible when accessing the file system from a remote APP-VM. The overhead due to SELinux in the read test is less than $1 \%$, if the APP-VM and the FS-VM run on the same host, and about $2 \%$ otherwise.

We have executed the same benchmark test on a FS-VM when the A-VM protects the kernel integrity through introspection with a period of 1 second between each invocation of the checks. In this case, the overhead is about $7 \%$ with respect to the case where no consistency checks are applied.

\section{Related Works}

Currently, SELinux includes both types to allow a client to label files imported from a NFS server and rules to define relationships among these types. Thus, if an APP-VM uses SELinux, the security policy can define distinct types for the files imported from each distinct NFS server. [10] describes the architecture developed by NSA so that SELinux can work over NFS and implement security controls acting as a local file system mechanism. This architecture correctly handles file and process labels and takes access decisions according to those labels, but it requires SELinux to be installed both on the server side and on the client one. 


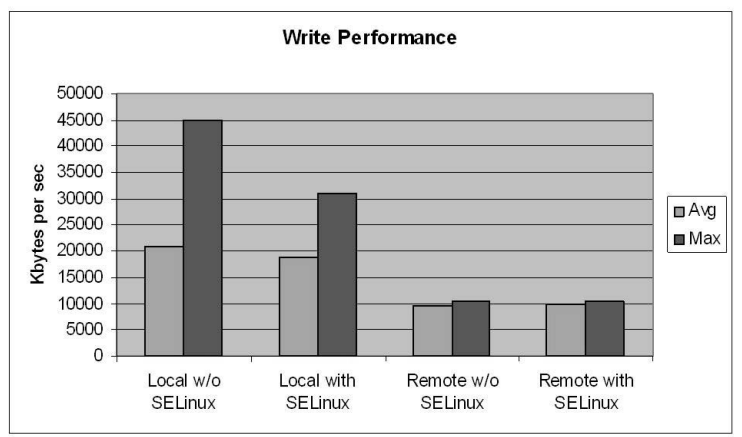

(a)

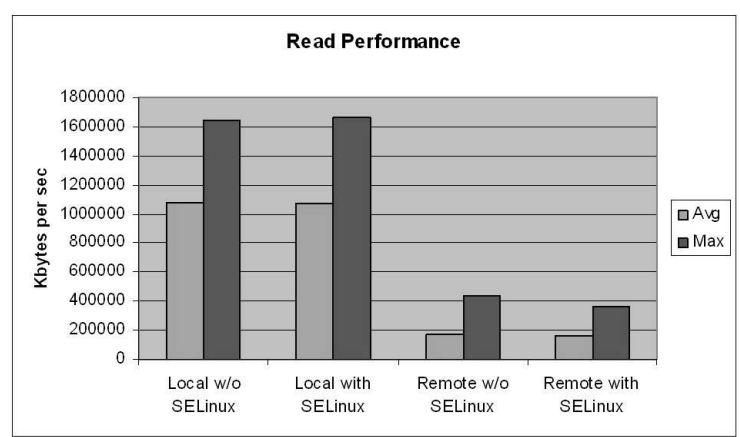

(b)

Figure 5. Write performance(a) and read performance(b).

Basically, the server gets the security context of the requesting process from the client, which adds it to the end of the RPC header, and it uses the context to take security decisions. The client gets and sets the security context of files accessed through NFS. This technique introduces several constraints and uses untrusted NFS client user credentials as the basis for security policy decisions. Posix Access Control Lists (ACLs) [15] extend the basic set of read, write, execute permissions of a classical Unix system. Both NFSv3 and NFSv4 support ACLs as optional file attributes, but the problem is that several OSes and file systems do not support ACLs. sHype [24] is a hypervisor security architecture developed by the IBM Research Group, which leverages hypervisor capability to isolate malicious OSes from accessing other VMs. This project, based upon the Xen hypervisor, is focused on controlled resource sharing among VMs according to formal policies. This approach is alternative to discretionary controls that assume the users are acting in authorized way. sHype explicitly controls information flows during inter-VM communications. Besides, sharing is defined and enforced by MAC policies, such as Chinese Wall Policy and Simple Type Enforcement, which support a coarse-grained resource management. Vault [16] is a system that, even in case of malware, prevents the exposure of sensitive users information through a trusted component executed in a VM separated from the user VMs. Vault also defines the protocol to coordinate the interactions among the users, the trusted component and the remote server that requests the sensitive data.
Self-securing storage [27] enables storage devices to safeguard data even in case of an OS compromise because any software running on the system attached to the storage devices cannot disable the security mechanism. This requires separate software and hardware modules for the storage servers. [21] further extends this technology, by describing a storage-based IDS, embedded in an NFS server. This IDS discovers unexpected changes to a set of specified system files, binaries, through rule-based detection techniques. Since storage devices export a block-based interface, they have to rebuild file system structures from low level information. Similarly, [29] proposes a storage-based IDS that exploits VM technology to prevent attacks against the IDS. The virtual disks define and manage a sector-to-file mapping table so that intrusion detection can be applied at the sector level. Ventana [23] is a virtualization aware file system that exploits the benefits of virtual disks to add versioning, access control and disconnected features to a conventional distributed file system. Finally, SVFS [30, 31] is an architecture that stores sensitive files on distinct VMs dedicated to data storage. Each access to sensitive files is mediated by SVFS that enforces access control policy, so that file protection cannot be bypassed even if a guest VM is compromised. Moreover, SVFS uses a virtual RPC mechanism to optimize the data exchange among virtual machines on the same node. 


\section{Conclusion and Future Developments}

The focus of VSFS is on the enforcement of MAC policies on a shared storage to protect files accessed by a set of applications with different trust levels. The ability to securely identify each APP-VM represents an important contribution of this project because it enables a FS-VM to reliably associate a security context to each APP-VM according to the VM trust level. In this way, we do not need to trust any information received from the APP-VMs to authenticate them. The first implementation of VSFS also shows that the performance overhead due to the mandatory access controls may be fairly acceptable. Moreover, the adoption of anti-spoofing techniques and virtual machine introspection further increases the overall security because, on the one hand, each APP-VM can be securely authenticated, on the other one, VSFS achieves high assurance about the integrity of the FS-VM.

If a finer-grained security policy is required, then the FS-VM can use additional user credentials, such as the local user ID, to enforce the current policy. As an example, VSFS may label each user with a security context that is a function of both the IP address of the APP-VM and the local user ID to further reduce the privileges of every user of an APP-VM. In this way, VSFS would limit the protection domain of any user to a proper subset of the one of the APP-VM. In fact, since the local user ID is untrusted, this approach reduces the protection domain of each user. Another approach is to transparently include client side assurance on the user ID [12].

We plan to extend VSFS with additional security policies, such as Chinese Wall Policies [8] or timeconstraint access control policies. As an example, we could request that two processes cannot open a file concurrently, or that a process may read a file only after some time has been elapsed since the last time a process wrote on it. Another area of interest is a stronger integration between VSFS and Psyco-Virt, to build highly secure systems that cannot be easily compromised by rootkits and the like. We envision an architecture where virtual machine introspection provides assurance about the integrity of the kernel running inside the VMs, while VSFS guarantees that critical files, such as system binaries, configuration files, libraries [31], are shared in a protected way among the VMs. Lastly, we plan to introduce encrypted file systems and to integrate them with virtual private networks among the VMs on distinct physical nodes to take into account attacks to the physical interconnection structure.

\section{References}

[1] IOzone Filesystem Benchmark. http://www. iozone.org/.

[2] Netfilter/Iptables project. www. netfilter.org/.

[3] Security-Enhanced Linux. http://www.nsa. gov/selinux/.

[4] The User-mode Linux Kernel Home Page. http: //user-mode-linux. sourceforge.net/.

[5] VMware. http://www. vmware.com/.

[6] The Xen virtual machine monitor. http: //www.cl.cam.ac.uk/Research/SRG/ netos/xen/.

[7] F. Baiardi and D. Sgandurra. Building Trustworthy Intrusion Detection through VM Introspection. In Proc. The Third International Symposium on Information Assurance and Security (IAS'07), 2007.

[8] D. F. C. Brewer and M. J. Nash. The chinese wall security policy. In IEEE Symposium on Security and Privacy, pages 206-214, 1989.

[9] B. Callaghan, B. Pawlowski, and P. Staubach. NFS Version 3 Protocol Specification. RFC 1813 (Informational), June 1995.

[10] J. Carter. Implementing SELinux Support for NFS. http://www.nsa.gov/selinux/papers/ nfsv3 .pdf.

[11] B. Dragovic, K. Fraser, S. Hand, T. Harris, A. Ho, I. Pratt, A. Warfield, P. Barham, and R. Neugebauer. Xen and the art of virtualization. In Proceedings of the ACM Symposium on Operating Systems Principles, October 2003.

[12] M. Eisler, R. Schemers, and R. Srinivasan. Security Mechanism Independence in ONC RPC. Proceedings of the 6th USENIX UNIX Security Symposium, 1996.

[13] T. Garfinkel and M. Rosenblum. A virtual machine introspection based architecture for intrusion detection. In Proc. Network and Distributed Systems Security Symposium, February 2003.

[14] R. P. Goldberg. Survey of virtual machine research. IEEE Computer, 7(6):34-45, 1974.

[15] A. Grünbacher. POSIX Access Control Lists on Linux. In USENIX Annual Technical Conference, FREENIX Track, pages 259-272, 2003.

[16] P. Kwan and G. Durfee. Vault: Practical Uses of Virtual Machines for Protection of Sensitive User Data. Technical report, PARC Technical Report. 
[17] P. Loscocco and S. Smalley. Integrating flexible support for security policies into the linux operating system. In Proceedings of the FREENIX Track: 2001 USENIX Annual Technical Conference, pages 29-42, Berkeley, CA, USA, 2001. USENIX Association.

[18] P. A. Loscocco and S. D. Smalley. Meeting critical security objectives with security enhanced linux. In Proceedings of the 2001 Ottawa Linux Symposium., 2001.

[19] J. Morris. Networking in NSA security-enhanced Linux. Linux Journal, 2005(129):3, 2005.

[20] C. Odhner. Security in NFS Storage Networks. Technical report, Network Appliance, February 2005.

[21] A. Pennington, J. Strunk, J. Griffin, C. Soules, G. Goodson, and G. Ganger. Storage-Based Intrusion Detection: Watching Storage Activity for Suspicious Behavior. In Proc. of Usenix Security Symposium, 2003.

[22] N. L. Petroni, T. Fraser, J. Molina, and W. A. Arbaugh. Copilot - a coprocessor-based kernel runtime integrity monitor. In USENIX Security Symposium, pages 179-194, 2004.

[23] B. Pfaff, T. Garfinkel, and M. Rosenblum. Virtualization aware file systems: Getting beyond the limitations of virtual disks. pages 353-366, 2006.

[24] R. Sailer, E. Valdez, T. Jaeger, R. Perez, L. van Doorn, J. L. Griffin, and S. Berger. sHype: A secure hypervisor approach to trusted virtualized systems. IBM Research Report, 2005.

[25] J. H. Saltzer and M. D. Schroeder. The protection of information in computer systems. Proceedings of the IEEE, 63(9):1278-1308, 1975.

[26] S. Smalley, C. Vance, and W. Salamon. Implementing SELinux as a Linux security module. Nai labs report, NAI Labs, Dec 2001. Revised May 2006.

[27] J. D. Strunk, G. R. Goodson, M. L. Scheinholtz, C. A. Soules, and G. R. Ganger. Self-Securing Storage: Protecting Data in Compromised Systems. In Proc. of the 4th Symposium on Operating Design and Implementation (OSDI), 2000.

[28] C. Wright, C. Cowan, S. Smalley, J. Morris, and G. Kroah-Hartman. Linux Security Modules: General Security Support for the Linux Kernel. In Proceedings of the 11th USENIX Security Symposium, pages 17-31, Berkeley, CA, USA, 2002. USENIX Association.

[29] Y. Zhang, Y. Gu, H. Wang, and D. Wang. Virtualmachine-based intrusion detection on file-aware block level storage. In SBAC-PAD '06: Proceedings of the 18th International Symposium on Computer Architecture and High Performance Computing (SBAC-PAD'06), pages 185-192, Washington, DC, USA, 2006. IEEE Computer Society.
[30] X. Zhao, K. Borders, and A. Prakash. Svgrid: a secure virtual environment for untrusted grid applications. In MGC '05: Proceedings of the 3rd international workshop on Middleware for grid computing, pages 1-6, New York, NY, USA, 2005. ACM Press.

[31] X. Zhao, K. Borders, and A. Prakash. Towards protecting sensitive files in a compromised system. In SISW '05: Proceedings of the Third IEEE International Security in Storage Workshop (SISW'05), pages 21-28, Washington, DC, USA, 2005. IEEE Computer Society.

[32] N. Zhu and T. Chiueh. Design, implementation, and evaluation of repairable file service, 2003. 